\title{
Towards a Better Understanding of Disordered Gambling: Efficacy of Animal Paradigms in Modelling Aspects of Gambling Behaviour
}

\author{
Paul J. Cocker ${ }^{1}$. Catharine A. Winstanley ${ }^{1}$
}

Published online: 5 July 2015

(C) Springer International Publishing AG 2015

\begin{abstract}
The parallels between gambling and substance addiction are increasingly acknowledged; despite this, legislation surrounding gambling is being progressively relaxed and opportunities to gamble continue to increase. There are currently no dedicated treatment options for gambling disorder, and as such, it may come to represent a considerable public health concern. Gamblers represent a heterogeneous population; yet, deficits have been repeatedly observed in regard to impulse control and decision making. Additionally, gamblers typically demonstrate greater endorsement of irrational or distorted beliefs regarding gambling in comparison to healthy controls. In this review, we suggest that animal models may offer a valuable opportunity to elucidate the neurobiological basis underlying these three major areas of dysfunction. We present a number of rodent behavioural paradigms that indicate a somewhat common involvement of the dopamine $\mathrm{D}_{2}$-like receptor family. Yet, importantly, these tasks show that these behaviours appear to be both pharmacologically and neurologically dissociable. Use of these animal models could therefore theoretically inform more symptomdirected pharmacotherapies based on areas of dysfunction seen in individuals.
\end{abstract}

This article is part of the Topical Collection on Gambling

Paul J. Cocker

pcocker@psych.ubc.ca

Catharine A. Winstanley

cwinstanley@psych.ubc.ca

1 Present address: Department of Psychology, The University of British Columbia, 2215 Wesbrook Mall, Vancouver, BC V6T 1Z3, Canada
Keywords Disordered gambling · Animal models · Dopamine $\cdot$ Impulsivity $\cdot$ Near-miss $\cdot$ Decision making

\section{Introduction}

Gambling or wagering on uncertain outcomes has been reported throughout human history. Present in virtually every culture, gambling is undertaken and enjoyed by the majority of individuals within the society [1-3]. For most people, gambling is simply a harmless pastime, but for some, it can become a maladaptive compulsion with severe repercussions on an individuals' quality of life in a manner akin to drug or alcohol addiction [4]. The symptoms of such cases of gambling disorder (GD) are so similar to those used to diagnose drug addiction that GD was re-classified as an addiction, rather than an impulse control, disorder in the 5th edition of the Diagnostic and Statistical Manual [5]. Increasing access to gambling opportunities means that prevalence rates of GD are likely to rise [6,7]; yet, only limited success has been achieved with either pharmacological or behavioural treatments, and there are currently no dedicated treatment options [8-10]. A possible reason for this lack of success in developing appropriate therapeutics for GD may lie in the heterogeneity within gambling. Not only are there a multitude of different games within the gambling panoply, but gamblers themselves represent a diverse group [11•]. Whether there are common vulnerabilities that precede the development of GD is not currently clear, but there is little evidence to support any prototypical neurobiological dysfunction in individuals with GD, in the same way as is often reported in alcohol or substance addiction $[12,13]$.

Studies using non-human subjects such as rats have the potential to meaningfully develop our understanding about the pathogenesis of human disorders and inform novel 
treatments [14]. Although animal models cannot mimic GD per se, they can model aspects of gambling-related behaviour and thus make a critical contribution to our understanding of the cognitive processes underlying engagement in gambling. Considering different gambling behaviours as potentially subject to independent expression and regulation, rather than assuming a universal 'pro-gambling' phenotype from the outset, may be more appropriate when trying to elucidate risk factors for disorders like GD in which the etiology is complex and likely multifactorial; moreover, such an approach is in-line with emerging diagnostic frameworks $[15,16]$. To this end, we will focus here on three cognitive domains within which gamblers exhibit dysfunction, namely in regard to increased impulsivity [17-22], disadvantageous cost/benefit decision making [23-25] and increased endorsement of irrational beliefs or cognitive biases pertaining to gambling [22, 26, 27]. Although these three areas represent a broad swathe of behaviours, each is not only subjectively but objectively quantifiable, making it possible to design appropriate paradigms for use in non-humans subjects. For the sake of brevity, we will also restrict discussion to the use of laboratory rodents to model these behaviours, as rodent models have been used more widely for neural and pharmacological characterisation studies, enabling data to be evaluated across multiple experiments.

The majority of the paradigms developed to date utilise computer-controlled operant chambers that minimise interexperimenter variation and allow for multiple behavioural measurements as well as rigid parameter control through greater automation. All the tasks discussed here can be run in standard 5-hole operant chambers. These chambers contain an array of 5 response apertures on one wall, each fitted with an infrared beam capable of detecting nose-poke entries. Along the opposite wall, two retractable levers or other manipulanda can be installed, typically positioned on either side of a food tray into which sugar pellets are delivered via an external dispenser.

\section{Impulsivity}

Impulsivity can broadly be defined as acting or making decisions without appropriate forethought [28]. Although some level of impulsivity can be adaptive in both human and animal populations, high levels of impulsivity inevitably result in deleterious consequences and are associated with a wide range of behavioural disorders, including problem gambling [29, 30].

Factor analysis of self-report questionnaires, as well as analyses of intra-individual behavioural variation, indicates that impulsivity is a non-unitary construct. While there is still some debate over the most appropriate way to define and measure different aspects of impulsivity, the Barratt
Impulsivity Scale (BIS-11) remains one of the most commonly used assessment scale [31]. This metric typically identifies three sub-components of motor, non-planning and attentional impulsivity. Most of the established rodent models of impulsive behaviours have been designed to model one of these sub-types [32]. Previous reviews have dealt extensively with modelling differing forms of impulsivity in animal models [28, 33-35]. Therefore, we will limit our discussion to two commonly used rodent paradigms that have arguably the most face, construct and predictive validity. The first is the 5-choice serial reaction time task (5-CSRTT), which is loosely analogous to the continuous performance task (CPT) in humans, and which has recently been successfully back-translated into humans, further confirming its validity [36]. The CPT requires participants to scan a 5-digit sequence and respond only when that sequence matches a 'target' sequence. Errors of commission occur when the subject responds prematurely to a sequence that matches the target stimulus in all but the last number. To avoid such impulsive responses, subjects must therefore wait to fully identify the target sequence. Subjects with GD make more of these premature responses on the CPT than healthy controls, indicative of greater motor impulsivity [37].

Secondly, we will discuss a model of impulsive decision making known as delay discounting, in which impulsive choice is defined as the selection of a smaller immediately available reward over a larger delayed one and is thought to represent intolerance to delay of gratification. Various delaydiscounting tasks have been used widely in both humans and animals [38]. The size of the reward and/or the length of the delays are varied in order to generate a hyperbolic discounting curve. Steeper discounting curves i.e. increased preference for smaller-sooner rewards have been repeatedly shown in subjects with GD [20, 22, 39].

\section{5-Choice Serial Reaction Time Task}

The 5-CSRTT requires animals to scan the 5-hole array in order to accurately detect the brief illumination (typically $0.5 \mathrm{~s}$ ) of one of the apertures. The animal must make a 'nosepoke' response in the hole that was illuminated in order to gain food reward, thereby providing a measure of animals' visuospatial attention. Responses made prematurely, before the stimulus light is illuminated, generates an index of motor impulsivity [40].

The 5-CSRTT has been used extensively in numerous labs around the world, such that a comprehensive review of both the pharmacological and neurobiological regulation of task performance would be beyond the scope of this review to discuss fully [see 40 for review]. Yet, what does appear clear is that not only are various aspects of performance behaviourally and pharmacologically dissociable, but they also depend on distinct core neural loci. In specific regard to 
premature responses on the 5-CSRTT, amphetamine has particularly prominent and robust effects [41]. Amphetamine is a psychostimulant that affects multiple neurochemical systems, including those of the monoamines dopamine, serotonin and noradrenaline [42]. Amphetamine-induced elevations in premature responding have been independently replicated by numerous groups and can be attenuated by administration of both systemic and intra-accumbens dopaminergic antagonists, as well as selective ablation of dopaminergic terminals in the striatum [41]. Critically, similar elevations in impulsivity are also observed after administration of the selective dopamine reuptake inhibitor GBR12909, as well as other psychostimulants such as cocaine and methylphenidate [43]. Although serotonergic and noradrenergic compounds, such as the selective 5- $\mathrm{HT}_{2 \mathrm{C}}$ receptor antagonist SB242084 [44] and alpha-2 receptor antagonist yohimbine [45], also increase premature responding, neither serotonin- or noradrenaline-specific reuptake inhibitors mimic this action of amphetamine [46]. As such, it would appear that, in the most general terms, potentiation of dopaminergic signalling has a particularly prominent role to play in mediating this form of behavioural disinhibition.

In a similar manner to systemic pharmacological manipulations, differing cortico-striatal circuitry appears to subserve distinct aspects of 5-CSRTT performance. With regard to the prefrontal cortex (PFC), whereas accuracy of attentional performance appears to depend on the integrity of the prelimbic area $(\operatorname{PrL})$, lesions to either the anterior cingulate cortex (ACC) or infralimbic cortex (IL) selectively increase impulsive responding [47]. Elevations in both premature and perseverative responding have also been reported after lesions or inactivation of the orbitofrontal cortex (OFC) [48]. It should also be noted that lesions of the nucleus accumbens produced more selective impairments in impulse control, with increases in premature responding only observed on trials directly following an incorrect response [49].

\section{Delay Discounting}

Similar to human paradigms, rodent delay-discounting tasks involve the choice of an immediately available small reward or a larger reward delivered after a delay. Operant tasks can be divided broadly into those which use a fixed set of delays and rewards that change either within or between sessions, or those in which either the size of the rewards or the delay to the delivery of the larger reward is varied on a trial-by-trial basis depending on the animals' choice on the previous trial [see 50 for review]. Perhaps the most widely used methodology is that based on Evenden and Ryan's original paradigm which was developed specifically to enable the efficacy of pharmacological challenges to be assessed and therefore incorporates standard within-session shifts in delay [51]. This method has the advantage of simplicity [see 52 for potential confounds present in adjusting delay/amount tasks], although it may not allow for a true assessment of delay-sensitivity independent of subjective reward evaluation [see 53 for multiplicative hyperbolic discounting model discussion]. In the Evenden and Ryan model, the animal chooses between a small reward (typically 1 sugar pellet) delivered immediately or a larger reward (typically 4 pellets) that is delivered after a delay. This delay increases in a step-wise session across the blocks, for example from 0 to $10,20,40$ and $60 \mathrm{~s}$. All trials are of equivalent length, such that selection of the larger reward always results in the most reward at any point in the session.

Similar to premature responding on the 5-CSRTT, impulsive choice on delay-discounting tasks appears sensitive to pharmacological agents that potentiate the effects of dopamine. Yet, in contrast to the increased premature responding reliably observed on the 5-CSRTT, most papers report that administration of amphetamine decreases impulsive choice on delay-discounting tasks, increasing choice of the larger but delayed reward [see 34 for discussion]. This increase can be blocked with prior administration of a $D_{2}$ but not a $D_{1}$ antagonist - moreover, the effects of amphetamine are mimicked by GBR12909 but not the norepinephrine reuptake inhibitor desipramine [54].

Although broadly similar, there are differences in the cortico-striatal circuits that mediate behaviour on the 5CSRTT and delay discounting. For instance, ACC lesions increase motor impulsivity on the 5-CSRTT but do not affect impulsive decision making on delay discounting [55]. Likewise, excitotoxic lesions to the OFC increase perseverative and premature responding on the 5-CSRTT [56] but can produce bidirectional effects on impulsive decision making on delay discounting [57], dependent upon task contingencies and subjective baseline behaviour [see 58 and 59 for discussion]. In contrast to these differences, both the 5-CSRTT and delay discounting are affected by lesions to the PrL cortex, whereby animals are less able to respond appropriately to task contingencies but are not specifically more or less impulsive $[47,55]$. Similarly, impulsivity on both tasks is increased following lesions to the nucleus accumbens $[49,55]$ and ventral hippocampus [60, 61].

\section{Summary}

Both the 5-CSRTT and delay discounting appear to be subserved by fronto-striatal circuits as well as the ventral hippocampus, and impulsive responses on both tasks can be modulated by dopaminergic activity. Yet, beyond these superficial similarities, there are task-specific differences that suggest caution when extrapolating from one metric of a behavioural construct to another and further clarify the need for a comprehensive characterisation of differing forms of impulsivity within different forms of gambling. 
Delay discounting is arguably one of the most wellcharacterised impulsivity measures in human gamblers [62], and as such, tasks in rodents may offer an opportunity to elucidate the neural circuitry that might underlie this form of impulsive choice.

The 5-CSRTT not only allows the measurement of prepotent impulsive responses, but also provides an index of compulsive action - when animals continue to respond in apertures even after food delivery has been signalled [40, 63]. Compulsivity and impulsivity are often viewed as diametrically opposed, but there is growing recognition that they may be dissociable but related constructs [64], particularly in regard to GD [65] where they may underlie differing forms of dysfunctional gambling behaviour. Lastly, the 5-CSRTT is able to parametrise aspects of attention and motivation, cognitive functions that are impaired in subjects with ADHD. Given the high concurrent morbidity rates between ADHD and gambling [66], the 5-CSRTT may represent a compelling paradigm with which to investigate multiple aspects of dysfunction typically seen within GD.

\section{Decision-Making Deficits}

Problem gambling could be conceptualised as increased risky financial decision making. Indeed, human gamblers consistently demonstrate impaired performance on tasks measuring cost/benefit decision making under both risk and ambiguity, such as the Cambridge gambling task [19], game of dice task [25] and the Iowa Gambling Task (IGT) [67], deficits that cannot exclusively be accounted for by increased impulsivity or deficits in cognitive ability [67]. Amongst the laboratory tasks used to probe aberrant decision making, the IGT has been the most widely used and several rodent analogues have been developed. The IGT requires participants to choose cards from four decks in order to accumulate points. Two decks are advantageous, associated with smaller immediate gains but also smaller losses. In contrast, the two disadvantages decks are associated with larger gains but also disproportionately larger long-term losses. Ergo, the optimal strategy is to avoid the tempting 'high-risk high-reward' options and instead choose from the less risky decks and steadily accrue smaller amounts [68]. Persistent choice of the former, disadvantageous decks is commonly observed in both gambling and substance use disorder $[67,69,70]$, further indicating that addictive disorders may share a common etiology. Yet, whether aberrant decision making on these tasks arises subsequently or antecedent to chronic exposure to gambling games is unclear. Animal models of such decision making may aid in elucidating vulnerabilities to such cognitive perturbations and the relationship of these deficits to other areas of dysfunction such as impulsivity. Although multiple versions of a rodent IGT have been developed with differing strengths and weaknesses [see 71 for discussion], the rat gambling task (rGT) has been the most widely adopted.

\section{The Rat Gambling Task}

The rGT, consistent with the IGT, requires animals to choose between four options with established contingencies. Two options are disadvantageous - associated with larger gains (food reward) but more frequent and larger punishments (time-out periods); whereas, the other two options are advantageousassociated with smaller gains but smaller and less frequent punishments. Animals have $30 \mathrm{~min}$ to maximise their 'earnings'; therefore, these time-out periods reduce the opportunity to earn reward. Thus, analogous to the human version, animals must learn to choose the low-reward low-punishment 'decks' more often than the superficially alluring but ultimately disadvantageous ones [72].

Results with the rGT demonstrate that animals show a very similar pattern of behavioural responses to humans playing the IGT, with selection of the tempting high-risk options declining with increased experience with the contingencies, resulting in a clear preference for options linked to smaller but safer rewards [72]. Optimal decision making on the rGT is modulated by multiple pharmacological systems. Systemic administration of amphetamine or the 5- $\mathrm{HT}_{1 \mathrm{~A}}$ agonist $8-\mathrm{OH}-$ DPAT both resulted in similar impairments with animals selecting the optimal options less following administration of either compound. In contrast, the dopamine $\mathrm{D}_{2}$-like receptor antagonist eticlopride improved optimal choice behaviour [72]. Despite the similar effects of 8-OH-DPAT and amphetamine, subsequent investigations have indicated that the effects of amphetamine on choice do not appear to be due to its actions at a single monoamine transmitter but rather the additive effects on multiple systems. Systemic administration of reuptake inhibitors for serotonin, dopamine or norepinephrine in isolation produces only mild effects on task performance. In contrast, any combination of two reuptake inhibitors significantly impaired behaviour on the rGT in a similar manner to that observed following systemic amphetamine [73]. Interestingly, amphetamine's effects on choice behaviour appear to be somewhat dissociable from those on motor impulsivity. The rGT, in a similar manner to the 5-CSRTT, also measures premature responding. Consistent with pharmacological data from the 5-CSRTT discussed above, motor impulsivity is increased following the administration of amphetamine, an effect which appears contingent on increasing extra-synaptic levels of dopamine, but not serotonin or norepinephrine [73, 74]. These data imply that risky decision making and impulsivity are dissociable. However, a recent meta-analysis revealed that at a population level, motor impulsivity on the rGT and choice of the risky disadvantageous options are well correlated. Thus, although these two constructs may be pharmacologically dissociable, indicating discrete traits, they may 
have synergistic effects on one another when both are present to a greater degree [see 75 for discussion].

Human performance on the IGT has been demonstrated to rely on the integrity of brain regions that underlie the formation and maintenance of addictive behaviours, particularly the ventromedial prefrontal cortex (VMPFC) and amygdala [68, 76-78]. Hypoactivity of prefrontal regions is one of the most oft-reported findings from imaging studies of subjects with GD, and the decisionmaking profile of problem gamblers on the IGT is characteristic of those with focal lesions to prefrontal cortical regions [67, 73, 79]. Similarly, animals performing the rGT show impairments following inactivations to the basolateral amygdala or OFC and disconnection of these two areas also retards learning of the optimal task strategy $[80,81]$. Lesions to the prefrontal cortex can also reduce preference for the advantageous options [82]. Lastly, recent work has highlighted a key role for the agranular insula and medial prefrontal cortex in mediating choice behaviour on the rGT, as either inactivations or lesions to these regions decreased optimal decision making by promoting the most risk-averse strategy [83]. These data are congruent with the notion that increased insula activity may facilitate detrimental gambling-related decision making, as patients with focal lesions to the insula cortex appear immune to various gambling-related cognitions [84].

\section{Summary}

Data from the rGT suggests decision-making circuitry is relatively well conserved across species, and tasks such as the rGT may offer an opportunity to elucidate circuits that underlie dysfunctional decision making. Furthermore, these tasks can also explore the relationship between constructs such as impulsivity and impaired decision making and how these constructs interact. The data derived from the rGT thus far suggests that, while $\mathrm{D}_{2}$-like receptors may be important for modulating impulsive decision making under risk, multiple pharmacological systems appear to underlie perturbations in cost/benefit decision making. The complex relationship between impulsivity and risky decision making alluded to above is intriguing and leads to the suggestion that examining traits as a continuum, rather than a dichotomy, even within nonclinical populations may be informative. Moreover, these individual differences are clearly demonstrable across species and are worth examining further within animal models [75]. Of particular interest, in this regard, is the recent translational study using the rGT to confirm the genetic association with $\mathrm{D}_{3}$ receptor expression in the CNS and poor decision making from a range of addiction-related genes [85].

\section{Cognitive Biases and Distortions}

Cognitive accounts of gambling claim that one of the primary reasons for the formation and maintenance of problem gambling is the presence of distorted or irrational beliefs concerning gambling outcomes. The presence of these perturbations is used to probe the severity of GD [86-88], and their continued presence following intervention predicts relapse [89]. Although there are a multitude of these cognitive distortions in relation to gambling, one that has received considerable attention is the 'near-miss' effect. Near-misses are unsuccessful outcomes that are proximal to a win (such as matching two items out of three on a slot machine payline). Despite being subjectively aversive, near-misses foster the belief of personal control and potentiate the illusion that a win is imminent [90]. Near-misses, although present in multiple forms of gambling, are particularly prominent in slot machines and have been alleged to contribute towards the virulence with which slot machines foster problematic gambling [91-93]. Imaging studies indicate that despite the lack of objective utility conveyed by near-miss trials, they are experienced as being more similar to winning outcomes, as both wins and near-misses recruit reward-related circuitry [94, 95]. Susceptibility to the 'rewarding' aspects of near-misses has been proposed to confer vulnerability to the development of GD [96 but see 97]. Slot machines are becoming increasingly complex but essentially rely on conditional discrimination and as such should be imitable using animal models. Indeed, two paradigms have been developed for use with rodents [98, 99], and of these, the rodent slot machine task (rSMT) has generated a greater amount of data.

\section{Rodent Slot Machine Task}

The rSMT requires animals to respond to 3 flashing lights roughly analogous to the wheels of a slot machine before choosing between two levers. One lever ('collect' lever) gives food reward on winning trials (when all 3 lights are illuminated) but a 10 -s time-out punishment on non-winning trials (when any of the 3 lights fails to illuminate); whereas, the other lever ('roll' lever) allows the animal to start a new trial straight away.

Animals clearly differentiate win trials from most loss trials, responding on the collect lever on $100 \%$ of win trials, but only $\sim 15-35 \%$ of the time on loss trials in which only 1 or 0 lights were illuminated. Hence, it is possible to infer that rats did not reliably associate such loss trials with the possibility of reward. In contrast, animals responded on the collect lever on $50-80 \%$ of loss trials when 2 lights were illuminated [99], indicating these types of trials were treated as more similar to wins than losses, and animals behaved as if these stimuli were predictive of available reward. As such, this behavioural 
pattern strongly resembles the near-miss effect observed in human subjects.

In human gamblers, amphetamine increases the desire to gamble, an effect which appears contingent on $\mathrm{D}_{2}$ receptors $[100,101]$. Similarly, amphetamine augments animals reward expectancy on the rSMT, an effect that is dependent on the dopamine $\mathrm{D}_{2}$-like receptor family, as the $\mathrm{D}_{2}$-like agonist quinpirole also increases animals' erroneous expectation of reward on non-winning trials $[99,102 \bullet]$. The $\mathrm{D}_{2}$-like receptor family contains $D_{2}, D_{3}$ and $D_{4}$ receptors, and the deleterious effects observed on the rSMT following quinpirole administration are predominantly mediated by the selective activation of $\mathrm{D}_{4}$ receptors. $\mathrm{A} \mathrm{D}_{4}$ agonist produced similar impairments to quinpirole, and a $\mathrm{D}_{4}$ antagonist produced a concomitant improvement in optimal lever choice and could attenuate quinpirole's negative effects [102•]. $\mathrm{D}_{4}$ receptors are preferentially located within prefrontal regions, including areas that have been implicated in problem gambling such as the anterior cingulate cortex and insula cortex [84, 94]. Preliminary data indicates that the ACC is critical for optimal performance on the rSMT, as inactivations increase erroneous collect responses. Furthermore, infusion of the $\mathrm{D}_{4}$ agonist PD168077 into the ACC also resulted in impairments, indicating a crucial role for these receptors in controlling responsivity to salient reward-related stimuli [103].

\section{Summary}

The presence of cognitive biases and beliefs may be vitally important in the formation and maintenance of GD. Tasks such as the rSMT demonstrate that rats, like humans, are susceptible to the putative winning signals conveyed within nonwinning trials. Moreover, the efficacy of these reward-related cues to facilitate the representation of reward appears contingent on comparable brain regions. Therefore, such tasks offer an invaluable platform to develop novel pharmacotherapies for disordered slot machine play. In support of such a conclusion, data from the rSMT thus far indicates that $\mathrm{D}_{4}$ receptor modulation can manipulate spurious responses to win-like stimuli. $\mathrm{D}_{4}$ receptors are located on both pyramidal neurons and local GABAergic interneurons and have been implicated in maintaining homeostatic activity levels within the PFC [104, 105]. Consequently, modulation of these receptors may foster a more adaptive interpretation of subjectively salient reward-related stimuli during gambling [106].

\section{Conclusions}

This review has touched upon some of the many animal tasks available that can mimic aspects of dysfunction seen in human gamblers. Animal models have the benefit of being relatively cost-effective, as compared to human imaging or clinical trials, and also offer a degree of control in regard to pharmacological and neuroanatomical specificity that would be impossible to achieve with human research [107]. Multiple transmitter systems and neural loci appear to be critically involved in mediating different aspects of gambling-related behaviour and decision making; yet, the dopamine $\mathrm{D}_{2}$ receptor family appears to represent a common target. There are however critical differences, indicating that tasks with clearly defined operational constructs may aid in delineating multiple dysfunctions underlying conditions such as GD. Tasks that aim to mimic aspects of decision making where there is established homology between humans and animals, such as those described here, may be more efficacious than attempting to model an entire disease state [107]. The overlap between human analogues and the tasks we have discussed are suggestive of underlying similarities in both neuroanatomy and pharmacology, which is encouraging in the use of animal methods to develop novel pharmacotherapies. Ultimately, the key may be a more effective delineation of types of gamblers, as viewing gamblers as a homogeneous group has not been productive at making meaningful strides towards developing treatments. Elucidating primary areas of dysfunction within different sub-groups may allow for a more targeted approach to treating symptoms as oppose to a disease, and the tools to model these aspects of dysfunction in animals are available.

Acknowledgments PJC is funded through a graduate student award from Parkinson's society Canada. CAW receives salary support through the Michael Smith Foundation for Health Research and the Canadian Institute for Health Research (CIHR) New Investigator Program.

\section{Compliance with Ethics Guidelines}

Conflict of Interest Paul J. Cocker and Catharine A. Winstanley declare that they have no conflict of interest.

Catherine A. Winstanley has previously consulted for Shire on an unrelated matter.

Human and Animal Rights and Informed Consent Animal studies were done by the authors (but no human studies).

This article does not contain any studies with human subjects performed by any of the authors.

With regard to the authors' research cited in this paper, all institutional and national guidelines for the care and use of laboratory animals were followed.

\section{References}

Papers of particular interest, published recently, have been highlighted as:

- Of importance

1. Wardle H, Moody A., Spence S, Orford J, Volberg R, Jotangia D, Griffths M, Hussey D, Dobbie F. British gambling prevalence survey 2010. The Gambling Commission, 2010. 
2. Azmier JJ. Canadian gambling behaviour and attitudes. Gambling in Canada Research report 2000.

3. Gerstein D, Hoffman J, Larison C, Engelam L, Murphy S, Palmer A, Chuchro L, Toce M, Johnson R, Buie T, Hill MA. Gambling impact and behavior study. Report to the National Gambling Impact Study Commission, 1999.

4. Potenza MN. Should addictive disorders include non-substancerelated conditions? Addiction. 2006;101 Suppl 1:142-51.

5. American Psychiatric Association. and American Psychiatric Association. DSM-5 Task Force., Diagnostic and statistical manual of mental disorders: DSM-5. 5th ed. Washington, D.C: American Psychiatric Association. xliv; 2013. 947 p.

6. Griffiths M. Gambling technologies: prospects for problem gambling. J Gambl Stud. 1999;15(3):265-83.

7. Pearce $\mathrm{J}$ et al. A national study of neighborhood access to gambling opportunities and individual gambling behaviour. J Epidemiol Community Health. 2008;62(10):862-8.

8. Grant JE, Kim SW. Medication management of pathological gambling. Minn Med. 2006;89(9):44-8.

9. Grant JE, Odlaug BL, Schreiber LR. Pharmacological treatments in pathological gambling. Br J Clin Pharmacol. 2012.

10. Ladouceur $\mathrm{R}$ et al. Cognitive treatment of pathological gambling. J Nerv Ment Dis. 2001;189(11):774-80.

11. Petry NM. A comparison of treatment-seeking pathological gamblers based on preferred gambling activity. Addiction. 2003;98(5): 645-55. Concise review detailing recent neuroimaging work in relation to gambling.

12. Limbrick-Oldfield EH, van Holst RJ, Clark L. Fronto-striatal dysregulation in drug addiction and pathological gambling: consistent inconsistencies? Neuroimage Clin. 2013;2:385-93.

13. Volkow ND et al. Imaging dopamine's role in drug abuse and addiction. Neuropharmacology. 2009;56 Suppl 1:3-8.

14. Potenza MN. The importance of animal models of decision making, gambling, and related behaviors: implications for translational research in addiction. Neuropsychopharmacology. 2009;34(13): 2623-4.

15. Kirmayer LJ, Crafa D. What kind of science for psychiatry? Frontiers in Human Neuroscience, 2014. 8.

16. Morris SE, Cuthbert BN. Research domain criteria: cognitive systems, neural circuits, and dimensions of behavior. Dialogues Clin Neurosci. 2012;14(1):29-37.

17. Potenza MN. Impulsivity and compulsivity in pathological gambling and obsessive-compulsive disorder. Rev Bras Psiquiatr. 2007;29(2):105-6.

18. Rodriguez-Jimenez R et al. Impulsivity and sustained attention in pathological gamblers: influence of childhood ADHD history. J Gambl Stud. 2006;22(4):451-61.

19. Lawrence AJ et al. Problem gamblers share deficits in impulsive decision-making with alcohol-dependent individuals. Addiction. 2009;104(6):1006-15.

20. Dixon MR, Marley J, Jacobs EA. Delay discounting by pathological gamblers. J Appl Behav Anal. 2003;36(4):449-58.

21. Petry NM. Pathological gamblers, with and without substance use disorders, discount delayed rewards at high rates. J Abnorm Psychol. 2001;110(3):482-7.

22. Michalczuk R et al. Impulsivity and cognitive distortions in pathological gamblers attending the UK National Problem Gambling Clinic: a preliminary report. Psychol Med. 2011;41(12):2625-35.

23. Linnet $\mathrm{J}$ et al. Dopamine release in ventral striatum during Iowa Gambling Task performance is associated with increased excitement levels in pathological gambling. Addiction. 2011;106(2): 383-90.

24. Goudriaan AE et al. Decision making in pathological gambling: a comparison between pathological gamblers, alcohol dependents, persons with Tourette syndrome, and normal controls. Brain Res Cogn Brain Res. 2005;23(1):137-51.
25. Brand $\mathrm{M}$ et al. Decision-making impairments in patients with pathological gambling. Psychiatry Res. 2005;133(1):91-9.

26. Billieux $\mathrm{J}$ et al. Trait gambling cognitions predict near-miss experiences and persistence in laboratory slot machine gambling. Br J Psychol. 2012;103:412-27.

27. Gaboury A, Ladouceur R. Erroneous perceptions and gambling. J Soc Behav Personal. 1989;4(4):411-20.

28. Winstanley CA, Eagle DM, Robbins TW. Behavioral models of impulsivity in relation to ADHD: translation between clinical and preclinical studies. Clin Psychol Rev. 2006;26(4):379-95.

29. Verdejo-Garcia A, Lawrence AJ, Clark L. Impulsivity as a vulnerability marker for substance-use disorders: review of findings from high-risk research, problem gamblers and genetic association studies. Neurosci Biobehav Rev. 2008;32(4):777-810.

30. Chamberlain SR, Sahakian BJ. The neuropsychiatry of impulsivity. Curr Opin Psychiatry. 2007;20(3):255-61.

31. Patton JH, Stanford MS, Barratt ES. Factor structure of the Barratt impulsiveness scale. J Clin Psychol. 1995;51(6):768-74.

32. Evenden JL. Varieties of impulsivity. Psychopharmacol (Berl). 1999;146(4):348-61.

33. Pattij T, Vanderschuren LJ. The neuropharmacology of impulsive behaviour. Trends Pharmacol Sci. 2008;29(4):192-9.

34. Winstanley CA. The utility of rat models of impulsivity in developing pharmacotherapies for impulse control disorders. Br J Pharmacol. 2011;164(4):1301-21. Provides detailed discussion of neurological and pharmacological manipulations on animal models of impulsivity.

35. Eagle DM, Bari A, Robbins TW. The neuropsychopharmacology of action inhibition: cross-species translation of the stop-signal and go/no-go tasks. Psychopharmacol (Berl). 2008;199(3):439-56.

36. Young JW et al. Reverse translation of the rodent 5C-CPT reveals that the impaired attention of people with schizophrenia is similar to scopolamine-induced deficits in mice. Transl Psychiatry. 2013;3, e324.

37. Kertzman $\mathrm{S}$ et al. Go-no-go performance in pathological gamblers. Psychiatry Res. 2008;161(1):1-10.

38. Ainslie G. Specious reward: a behavioral theory of impulsiveness and impulse control. Psychol Bull. 1975;82(4):463-96.

39. Alessi SM, Petry NM. Pathological gambling severity is associated with impulsivity in a delay discounting procedure. Behav Process. 2003;64(3):345-54.

40. Robbins TW. The 5-choice serial reaction time task: behavioural pharmacology and functional neurochemistry. Psychopharmacology. 2002;163(3-4):362-80.

41. Cole BJ, Robbins TW. Amphetamine impairs the discriminative performance of rats with dorsal noradrenergic bundle lesions on a 5-choice serial reaction time task: new evidence for central dopaminergic-noradrenergic interactions. Psychopharmacol (Berl). 1987;91(4):458-66.

42. Sulzer D et al. Mechanisms of neurotransmitter release by amphetamines: a review. Prog Neurobiol. 2005;75(6):406-33.

43. van Gaalen $\mathrm{MM}$ et al. Behavioral disinhibition requires dopamine receptor activation. Psychopharmacol (Berl). 2006;187(1):73-85.

44. Winstanley CA et al. 5-HT2A and 5-HT2C receptor antagonists have opposing effects on a measure of impulsivity: interactions with global 5-HT depletion. Psychopharmacol (Berl). 2004;176(3-4):376-85.

45. Sun $\mathrm{H}$ et al. Yohimbine increases impulsivity through activation of cAMP response element binding in the orbitofrontal cortex. Biol Psychiatry. 2010;67(7):649-56.

46. Baarendse PJ, Vanderschuren LJ. Dissociable effects of monoamine reuptake inhibitors on distinct forms of impulsive behavior in rats. Psychopharmacol (Berl). 2012;219(2):313-26.

47. Muir JL, Everitt BJ, Robbins TW. The cerebral cortex of the rat and visual attentional function: dissociable effects of mediofrontal, cingulate, anterior dorsolateral, and parietal cortex lesions on a 
five-choice serial reaction time task. Cereb Cortex. 1996;6(3): 470-81.

48. Chudasama Y, Robbins TW. Dissociable contributions of the orbitofrontal and infralimbic cortex to Pavlovian autoshaping and discrimination reversal learning: further evidence for the functional heterogeneity of the rodent frontal cortex. J Neurosci. 2003;23(25):8771-80.

49. Christakou A, Robbins TW, Everitt BJ. Prefrontal cortical-ventral striatal interactions involved in affective modulation of attentional performance: implications for corticostriatal circuit function. J Neurosci. 2004;24(4):773-80.

50. Hamilton KR et al. Choice impulsivity: definitions, measurement issues, and clinical implications. Pers Disord. 2015;6(2):182-98.

51. Evenden JL, Ryan CN. The pharmacology of impulsive behaviour in rats: the effects of drugs on response choice with varying delays of reinforcement. Psychopharmacol (Berl). 1996;128(2):161-70.

52. Cardinal RN et al. Local analysis of behaviour in the adjustingdelay task for assessing choice of delayed reinforcement. Neural Netw. 2002;15(4-6):617-34.

53. Ho MY et al. Theory and method in the quantitative analysis of "impulsive choice" behaviour: implications for psychopharmacology. Psychopharmacol (Berl). 1999;146(4):362-72.

54. van Gaalen MM et al. Critical involvement of dopaminergic neurotransmission in impulsive decision making. Biol Psychiatry. 2006;60(1):66-73.

55. Cardinal $\mathrm{RN}$ et al. Impulsive choice induced in rats by lesions of the nucleus accumbens core. Science. 2001;292(5526):2499-501.

56. Chudasama $\mathrm{Y}$ et al. Dissociable aspects of performance on the 5choice serial reaction time task following lesions of the dorsal anterior cingulate, infralimbic and orbitofrontal cortex in the rat: differential effects on selectivity, impulsivity and compulsivity (vol 146, pg 105, 2003). Behav Brain Res. 2004;152(2):453.

57. Winstanley CA et al. Contrasting roles of basolateral amygdala and orbitofrontal cortex in impulsive choice. J Neurosci. 2004;24(20):4718-22.

58. Zeeb FD, Floresco SB, Winstanley CA. Contributions of the orbitofrontal cortex to impulsive choice: interactions with basal levels of impulsivity, dopamine signalling, and reward-related cues. Psychopharmacol (Berl). 2010;211(1):87-98.

59. Floresco SB et al. Cortico-limbic-striatal circuits subserving different forms of cost-benefit decision making. Cogn Affect Behav Neurosci. 2008;8(4):375-89.

60. Abela AR et al. Inhibitory control deficits in rats with ventral hippocampal lesions. Cereb Cortex. 2013;23(6):1396-409.

61. Abela AR, Chudasama Y. Dissociable contributions of the ventral hippocampus and orbitofrontal cortex to decision-making with a delayed or uncertain outcome. Eur J Neurosci. 2013;37(4):640-7.

62. Miedl SF, Peters J, Buchel C. Altered neural reward representations in pathological gamblers revealed by delay and probability discounting. Arch Gen Psychiatry. 2012;69(2):177-86.

63. Carli $\mathrm{M}$ et al. Effects of lesions to ascending noradrenergic neurones on performance of a 5-choice serial reaction task in rats; implications for theories of dorsal noradrenergic bundle function based on selective attention and arousal. Behav Brain Res. 1983;9(3):361-80.

64. Grant JE, Kim SW. Brain circuitry of compulsivity and impulsivity. Cns Spectr. 2014;19(1):21-7.

65. Leeman RF, Potenza MN. Similarities and differences between pathological gambling and substance use disorders: a focus on impulsivity and compulsivity. Psychopharmacology. 2012;219(2): 469-90.

66. Crockford DN, el-Guebaly N. Psychiatric comorbidity in pathological gambling: a critical review. Can J Psychiatry. 1998;43(1): 43-50.

67. Cavedini $\mathrm{P}$ et al. Frontal lobe dysfunction in pathological gambling patients. Biol Psychiatry. 2002;51(4):334 41.
68. Bechara A et al. Insensitivity to future consequences following damage to human prefrontal cortex. Cognition. 1994;50(1-3):7-15.

69. Roca $\mathrm{M}$ et al. Executive functions in pathologic gamblers selected in an ecologic setting. Cogn Behav Neurol. 2008;21(1):1-4.

70. Power Y, Goodyear B, Crockford D. Neural correlates of pathological gamblers preference for immediate rewards during the Iowa gambling task: an fMRI study. J Gambl Stud. 2012;28(4): 623-36.

71. de Visser L et al. Rodent versions of the Iowa gambling task: opportunities and challenges for the understanding of decisionmaking. Front Neurosci. 2011;5:109.

72. Zeeb FD, Robbins TW, Winstanley CA. Serotonergic and dopaminergic modulation of gambling behavior as assessed using a novel rat gambling task. Neuropsychopharmacology. 2009;34(10):2329-43.

73. Baarendse PJ, Winstanley CA, Vanderschuren LJ. Simultaneous blockade of dopamine and noradrenaline reuptake promotes disadvantageous decision making in a rat gambling task. Psychopharmacol (Berl). 2013;225(3):719-31.

74. Zeeb FD, Wong AC, Winstanley CA. Differential effects of environmental enrichment, social-housing, and isolation-rearing on a rat gambling task: dissociations between impulsive action and risky decision-making. Psychopharmacol (Berl). 2013;225(2): 381-95.

75. Barrus MM et al. Disadvantageous decision-making on a rodent gambling task is associated with increased motor impulsivity in a population of male rats. J Psychiatry Neurosci. 2014;39(5): 140045.

76. Bechara A et al. Different contributions of the human amygdala and ventromedial prefrontal cortex to decision-making. J Neurosci. 1999;19(13):5473-81.

77. Jentsch JD, Taylor JR. Impulsivity resulting from frontostriatal dysfunction in drug abuse: implications for the control of behavior by reward-related stimuli. Psychopharmacol (Berl). 1999;146(4): 373-90.

78. Bechara A. Decision making, impulse control and loss of willpower to resist drugs: a neurocognitive perspective. Nat Neurosci. 2005;8(11):1458-63.

79. Bechara A et al. Decision-making deficits, linked to a dysfunctional ventromedial prefrontal cortex, revealed in alcohol and stimulant abusers. Neuropsychologia. 2001;39(4): 376-89.

80. Zeeb FD, Winstanley CA. Lesions of the basolateral amygdala and orbitofrontal cortex differentially affect acquisition and performance of a rodent gambling task. J Neurosci. 2011;31(6): 2197-204.

81. Zeeb FD, Winstanley CA. Functional disconnection of the orbitofrontal cortex and basolateral amygdala impairs acquisition of a rat gambling task and disrupts animals' ability to alter decision-making behavior after reinforcer devaluation. J Neurosci. 2013;33(15):6434-43.

82. Paine TA et al. Medial prefrontal cortex lesions impair decisionmaking on a rodent gambling task: reversal by D1 receptor antagonist administration. Behav Brain Res. 2013;243:247-54.

83. Pushparaj A, et al. Differential involvement of the agranular vs granular insular cortex in the acquisition and performance of choice behavior in a rodent gambling task. Neuropsychopharmacology, 2015.

84. Clark L et al. Damage to insula abolishes cognitive distortions during simulated gambling. Proc Natl Acad Sci U S A. 2014;111(16): 6098-103.

85. Lobo DS, et al. Addiction-related genes in gambling disorders: new insights from parallel human and pre-clinical models. Mol Psychiatry, 2014. 
86. Lesieur HR, Blume SB. The South Oaks Gambling Screen (SOGS): a new instrument for the identification of pathological gamblers. Am J Psychiatry. 1987;144(9):1184-8.

87. Raylu N, Oei TP. The Gambling Related Cognitions Scale (GRCS): development, confirmatory factor validation and psychometric properties. Addiction. 2004;99(6):757-69.

88. Steenbergh TA et al. Development and validation of the gamblers' beliefs questionnaire. Psychol Addict Behav. 2002;16(2):143-9.

89. Oei TPS, Gordon LM. Psychosocial factors related to gambling abstinence and relapse in members of gamblers anonymous. $\mathrm{J}$ Gambl Stud. 2008;24(1):91-105.

90. Reid RL. The psychology of the near miss. J Gambl Behav. 1986;2:32-9.

91. Breen RB, Zimmerman M. Rapid onset of pathological gambling in machine gamblers. J Gambl Stud. 2002;18(1):31-43.

92. Choliz M. Experimental analysis of the game in pathological gamblers: effect of the immediacy of the reward in slot machines. $\mathrm{J}$ Gambl Stud. 2010;26(2):249-56.

93. Dowling N, Smith D, Thomas T. Electronic gaming machines: are they the 'crack-cocaine' of gambling? Addiction. 2005;100(1): 33-45.

94. Clark L et al. Gambling near-misses enhance motivation to gamble and recruit win-related brain circuitry. Neuron. 2009;61(3): 481-90.

95. Chase HW, Clark L. Gambling severity predicts midbrain response to near-miss outcomes. J Neurosci. 2010;30(18):6180-7.

96. Habib R, Dixon MR. Neurobehavioral evidence for the 'nearmiss' effect in pathological gamblers. J Exp Anal Behav. 2010;93:313-28.

97. Shao R et al. Shifts in reinforcement signalling while playing slotmachines as a function of prior experience and impulsivity. Transl Psychiatry. 2013;3:e213.

98. Peters H, Hunt M, Harper D. An animal model of slot machine gambling: the effect of structural characteristics on response latency and persistence. J Gambl Stud. 2010;26(4):521-31.
99. Winstanley CA, Cocker PJ, Rogers RD. Dopamine modulates reward expectancy during performance of a slot machine task in rats: evidence for a 'near-miss' effect. Neuropsychopharmacology. 2011;36(5):913-25.

100. Zack M, Poulos CX. Amphetamine primes motivation to gamble and gambling-related semantic networks in problem gamblers. Neuropsychopharmacology. 2004;29(1):195-207.

101. Zack M, Poulos CX. A D2 antagonist enhances the rewarding and priming effects of a gambling episode in pathological gamblers. Neuropsychopharmacology. 2007;32(8):1678-86.

102. Cocker PJ, et al. A selective role for dopamine d receptors in modulating reward expectancy in a rodent slot machine task. Biol Psychiatry, 2013. Animal study suggesting a potential role for D4 receptor modulation in mediating dysfunctional reward expectancy during gambling.

103. Cocker PJ, Hosking JG, Winstanley CA. Dopamine D4 receptors in the anterior cingulate cortex appear to modulate reward expectancy on a rodent slot machine task, in The International College of Neuropsychopharmacology. 2014. p. P-01-010.

104. Mrzljak L et al. Localization of dopamine D4 receptors in GABAergic neurons of the primate brain. Nature. 1996;381(6579): 245-8.

105. Yuen EY, Zhong P, Yan Z. Homeostatic regulation of glutamatergic transmission by dopamine D4 receptors. Proc Natl Acad Sci U S A. 2010;107(51):22308-13.

106. Cocker PJ, Winstanley CA. Irrational beliefs, biases and gambling: exploring the role of animal models in elucidating vulnerabilities for the development of pathological gambling. Behav Brain Res. 2015;279C:259-73.

107. Davis KL. American College of Neuropsychopharmacology., Neuropsychopharmacology: the fifth generation of progress: an official publication of the American College of Neuropsychopharmacology. 2002, Philadelphia: Lippincott Williams \& Wilkins. xxi, 2010 p., 24 p. of plates. 